\title{
The Thirty Meter Telescope site testing system
}

Warren Skidmore, Matthias Schock, Andrei A. Tokovinin, George Djorgovski, Alistair R. Walker, et al.

Warren Skidmore, Matthias Schock, Andrei A. Tokovinin, George Djorgovski, Alistair R. Walker, Robert D. Blum, Tony Travouillon, Juan Seguel, Edison E. Bustos, David Walker, Joselina Vasquez, Paul E. Gillett, "The Thirty Meter Telescope site testing system," Proc. SPIE 5489, Ground-based Telescopes, (28 September 2004); doi: 10.1117/12.549384

Event: SPIE Astronomical Telescopes + Instrumentation, 2004, Glasgow, United Kingdom 


\title{
The Thirty Meter Telescope site testing system
}

\author{
Warren Skidmore $^{a}$, Matthias Schöck ${ }^{a}$, Andrei Tokovinin ${ }^{b}$, George Djorgovski ${ }^{a}$, Alistair \\ Walker $^{b}$, Robert Blum ${ }^{b}$, Tony Travouillon ${ }^{a}$, Juan Seguel ${ }^{b}$, Edison Bustos ${ }^{b}$, David Walker $^{b}$, \\ Joselino Vasquez $^{b}$, Paul Gillett ${ }^{c}$ \\ ${ }^{a}$ Caltech, Mail code 105-24, Pasadena, CA 91125-24, USA; \\ ${ }^{b}$ CTIO/AURA, Casilla 603, La Serena, Chile, S.A; \\ ${ }^{c}$ NOAO, 950 N. Cherry Avenue, Tucson, AZ 85719, USA
}

\begin{abstract}
The Thirty Meter Telescope (TMT) site testing team is developing a suite of instruments to measure the atmospheric and optical characteristics of candidate TMT sites. Identical sets of robotically operating instruments will be placed at each candidate site. The fully developed system will comprise of a combined MASS/DIMM, a SODAR, tower mounted thermal probes and a portable DIMM. These instruments have overlapping altitude coverage and provide a measure of the $\mathrm{C}_{\mathrm{n}}^{2}$ profile from the ground up with sufficient resolution to make conclusions about the ground layer and high altitude turbulence characteristics. The overlapping altitude coverage is essential to ensure consistency between these very different instruments. In addition to checking for consistency in the overlap regions, procedures are being used to cross check between instruments, i.e. the calculation of the isoplanatic angle from both the MASS and DIMM and that integrals of the $\mathrm{C}_{\mathrm{n}}^{2}$ profiles from the MASS, SODAR and $30 \mathrm{~m}$ tower give the same $\mathrm{r}_{0}$ values as measured by the DIMM.

We discuss a variation of the traditional DIMM system in which we employ a continuous drift mode readout technique giving a maximum of nearly 300 samples per second.

Findings of our major equipment testing campaigns and first field deployment are presented that demonstrate our progress in developing a rigorous approach to site testing.
\end{abstract}

Keywords: Site testing, DIMM, MASS, SODAR, microthermal probes, seeing, atmospheric turbulence

\section{INTRODUCTION}

\subsection{The aims of the TMT site testing campaign}

The TMT site testing campaign aims to gather appropriate optical, meteorological and atmospheric turbulence measurements to enable the quality of each candidate TMT site to be individually evaluated, i.e. to determine the optical turbulence as function of height from the ground to the upper atmosphere and to measure the basic weather properties and other relevant parameters of each site.

In order to achieve these aims we are carrying out an extensive comparison and calibration campaign of the basic instruments that we are using or are planning to use for site testing, namely DIMM, MASS, SODAR and microthermal towers.

Computational Fluid Dynamics (CFD) models are to be used to make accurate predictions of site properties and conditions before and after construction of the TMT (when the geography of the site will be changed from that present during site testing). We have also carried out a campaign involving all of the above instruments and simultaneous balloon measurements to gather data for evaluating and developing CFD models. The timescale for the TMT site decision is short and only allows for about two full years of simultaneous testing for all sites. Longer term meteorological data are available for each candidate site. CFD modeling will be used to give some guidance as to the expected long term quality of each site. ${ }^{6}$

This paper discusses the equipment and methods used or being developed to make measurements at remote sites.

Further author information:

E-mail: was@astro.caltech.edu 


\begin{tabular}{llll} 
Altitudes & Instrument & Resolution & Parameters \\
\hline $5 \mathrm{~m}-30 \mathrm{~m}$ & Tower & $5 \mathrm{~m}$ & $\mathrm{C}_{\mathrm{t}}^{2}, \mathrm{C}_{\mathrm{n}}^{2}$ \\
$20 \mathrm{~m}-1 \mathrm{~km}$ & SODAR & $10 \mathrm{~m}-50 \mathrm{~m}$ & $\mathrm{C}_{\mathrm{t}}^{2}, \mathrm{C}_{\mathrm{n}}^{2 \dagger}$ \\
$500 \mathrm{~m}-20 \mathrm{~km}$ & MASS & $1 \mathrm{~km}$ & $\mathrm{C}_{\mathrm{n}}^{2}, \theta_{0}, \tau_{0}^{*}$ \\
Integrated & DIMM & - & $\mathrm{r}_{0}, \theta_{0}, \tau_{0}^{*}$
\end{tabular}

Table 1. Planned scheme of atmospheric turbulence instrumentation. SODAR - SOnic Detection And Ranging, MASS - Multi-Aperture Scintillation Sensor, DIMM - Differential Image Motion Monitor. ( $\dagger$ - with an assumed temperature profile. * - using appropriate wind velocity information)

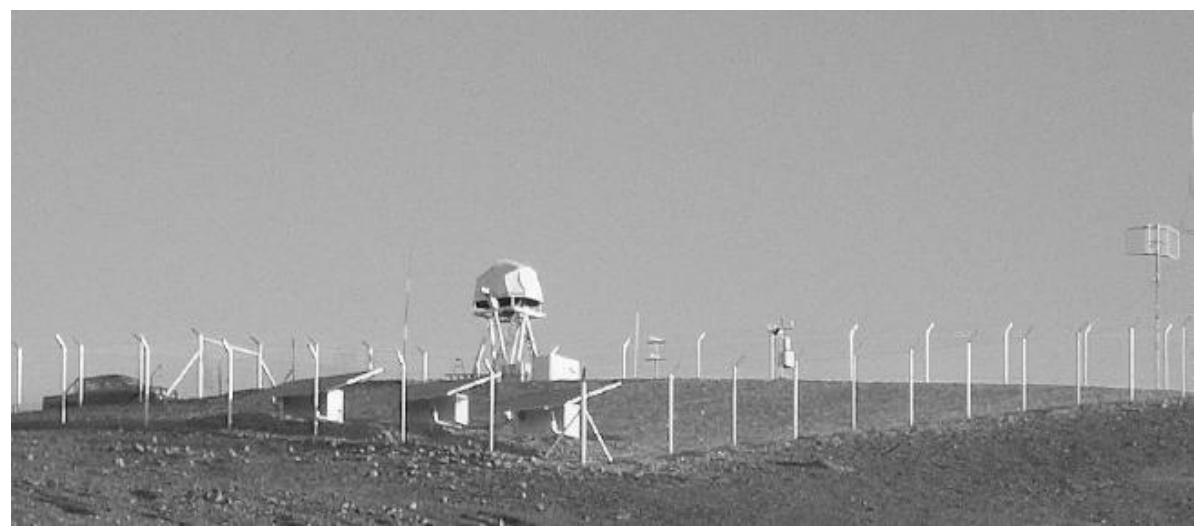

Figure 1. The first remote deployment of TMT site testing equipment. The telescope, weather stations, solar power system and microwave communication antenna are visible

\subsection{Status of the overall project}

The basic modular robotic site testing system has been developed. Each instrument is a self contained system. A supervisor computer controls the operation of the suite of instruments. One remote station consisting of a telescope with MASS/DIMM unit and a weather station is operating in northern Chile - Figure 1. This modular system can be expanded to include and control additional instrumentation as each new instrument is developed.

Beginning March 2004 (and still underway at the time of writing) we embarked on a large campaign at Cerro Tololo Inter-Americana Observatory (CTIO) to develop and characterise the full suite of equipment MASS/DIMM, SODARS and 30m tower. The aim of the campaign is to produce a calibrated self-consistent suite of instruments whose results are directly comparable to those made by different groups, i.e. we want to establish the standards for TMT site testing. In carrying out this campaign the full robotic system of instruments has been developed.

\section{THE EQUIPMENT BEING USED}

\subsection{Overview}

A combined Multi-Aperture Scintillation Sensor/Differential Image Motion Monitor (MASS/DIMM) system, SODAR, microthermal probes, fine wire thermocouples, sonic anemometers and portable DIMM system with overlapping altitude coverage provide a measure of the $\mathrm{C}_{\mathrm{n}}^{2}$ profile from the ground up with sufficient resolution to make conclusions about the ground layer characteristics and high altitude turbulence (see Table 1). The overlapping altitude coverage is essential to ensure consistent measurements of similar parameters between these very different instruments.

On remote sites the complete suite of instruments, all computers and the communication link are powered by a solar power system, internet connectivity is provided by a microwave or satellite network connection, see Figure 1. 


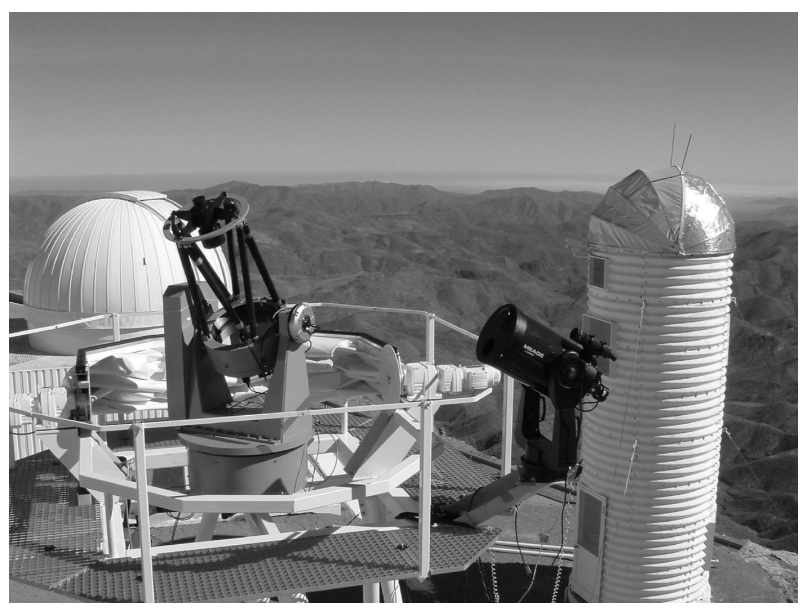

Figure 2. TMT MASS/DIMM \& portable DIMM atop a $6.5 \mathrm{~m}$ tower. The silver dome on the right covers the CTIO MASS/DIMM and the white dome on the left contains the CTIO portable MASS.

\subsection{Telescope mounted MASS/DIMM}

The heart of the site testing system is a $35 \mathrm{~cm} \mathrm{~F}=2.8 \mathrm{~m}$ robotic telescope atop a $6.5 \mathrm{~m}$ tower. The telescope and dome are designed to operate remotely for long periods of time and to withstand extremes of weather conditions. A combined MASS/DIMM unit (http://www.ctio.noao.edu/ atokovin/profiler/index.html) is fitted to the telescope. Pick off mirrors in the pupil image plane send light to the MASS unit and the two channels of a DIMM unit. The DIMM provides the basic measurement for assessing site quality, the value for the integrated seeing or $r_{0}$ from the height of the telescope to the edge of the atmosphere. The MASS is a profiling instrument ${ }^{4}$ that allows the optical turbulence profile $\left(\mathrm{C}_{n}^{2}(\mathrm{~h})\right)$ of the upper atmosphere to be reconstructed, the lower altitude limit for the MASS is about $500 \mathrm{~m}$. The integrated turbulence profile from the MASS allows a measure of the component of the seeing arising in the atmospheric layers above a few hundred meters. The scintillation measured in the DIMM is used to determine the isoplanatic angle as a check for the isoplanatic angle determined from the MASS measurements. ${ }^{2}$

\subsubsection{The modified DIMM method}

The normal DIMM method uses interlaced $5 \mathrm{~ms}$ and $10 \mathrm{~ms} 2 \mathrm{~d}$ images from which the differential image motion can be measured and a correction made to determine the seeing at zero exposure time. This method is far from efficient as the amount of dead time when reading out the CCD is many times longer than the exposure time. To improve this we have developed a new method of obtaining measurements of the differential image motion.

The new method uses the CCD detector in a continuous drift scan mode (see also DIMMWIT - B. O'Donovan et al., \& E. Seneta, B. O'Donovan, this proceedings). A small window surrounding the doubled stellar image is exposed and shifted down until the exposed region reaches the edge of the CCD. During final readout from the CCD the small $2 \mathrm{~d}$ image is binned vertically to reduce readout time. The effect is that we obtain a continuous time sequence of $1 \mathrm{~d}$ images with essentially zero deadtime. Our normal operating mode uses $200 \mathrm{~Hz}$ continuous sampling, the drift mode readout can operate at up to $300 \mathrm{~Hz}$ with our equipment.

The sequence of $1 \mathrm{~d}$ images is time binned in software to give measurements at $5 \mathrm{~ms}, 10 \mathrm{~ms}, 15 \mathrm{~ms}, 20 \mathrm{~ms}, 25 \mathrm{~ms}$ and $30 \mathrm{~ms}$. The differential image motion is measured for each exposure time. Two types of correction to zero exposure time are used: using the $5 \mathrm{~ms}$ and $10 \mathrm{~ms}$ measurements and a modified exponential extrapolation and a full six point exponential extrapolation. ${ }^{3}$

This new method has been validated using simulated data and real observations (see Section 3.2.1).

\subsection{0m Tower}

To investigate the turbulence in the lowest $30 \mathrm{~m}$ of the atmosphere, a $30 \mathrm{~m}$ tower populated with differential and absolute temperature sensors is employed. At present, the $30 \mathrm{~m}$ tower is populated at ten cosinusoidally spaced 


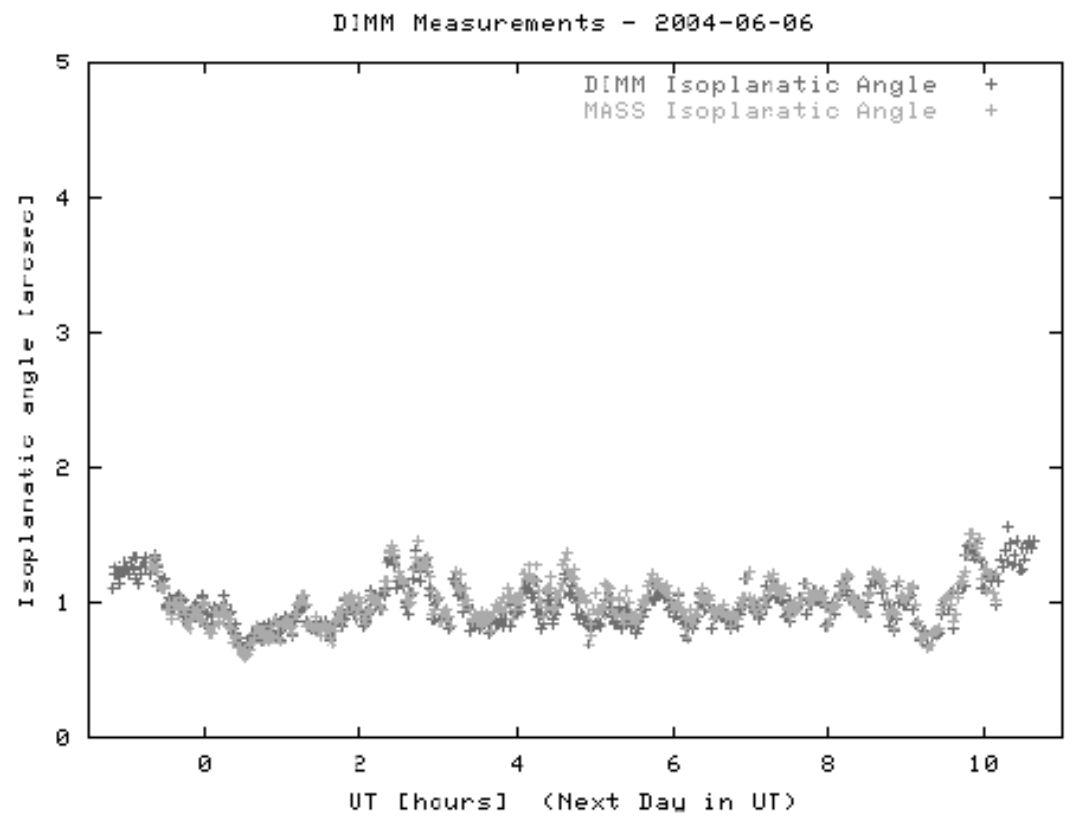

Figure 3. Isoplanatic angle determined from DIMM scintillation indices (black points) and measured by MASS (gray points). The gray points overlay the black points, showing a very good agreement

\begin{tabular}{lllll} 
Height $(\mathrm{m})$ & SA & MT & DT & AT \\
\hline 30.00 & $\mathrm{x}$ & $\mathrm{x}$ & $\mathrm{x}$ & $\mathrm{x}$ \\
25.31 & & $\mathrm{x}$ & $\mathrm{x}$ & $\mathrm{x}$ \\
20.72 & & & $\mathrm{x}$ & $\mathrm{x}$ \\
16.38 & & $\mathrm{x}$ & $\mathrm{x}$ & $\mathrm{x}$ \\
12.37 & $\mathrm{x}$ & $\mathrm{x}$ & $\mathrm{x}$ & $\mathrm{x}$ \\
8.79 & & & $\mathrm{x}$ & $\mathrm{x}$ \\
5.73 & & & $\mathrm{x}$ & $\mathrm{x}$ \\
3.27 & & $\mathrm{x}$ & $\mathrm{x}$ & $\mathrm{x}$ \\
1.47 & & $\mathrm{x}$ & $\mathrm{x}$ & $\mathrm{x}$ \\
0.37 & & & $\mathrm{x}$ & $\mathrm{x}$
\end{tabular}

Table 2. The arrangement of instrumentation on the $30 \mathrm{~m}$ tower. SA - sonic anemometer, MT - microthermal probes, DT - differenced thermocouples, AT - absolute temperature thermocouple. 


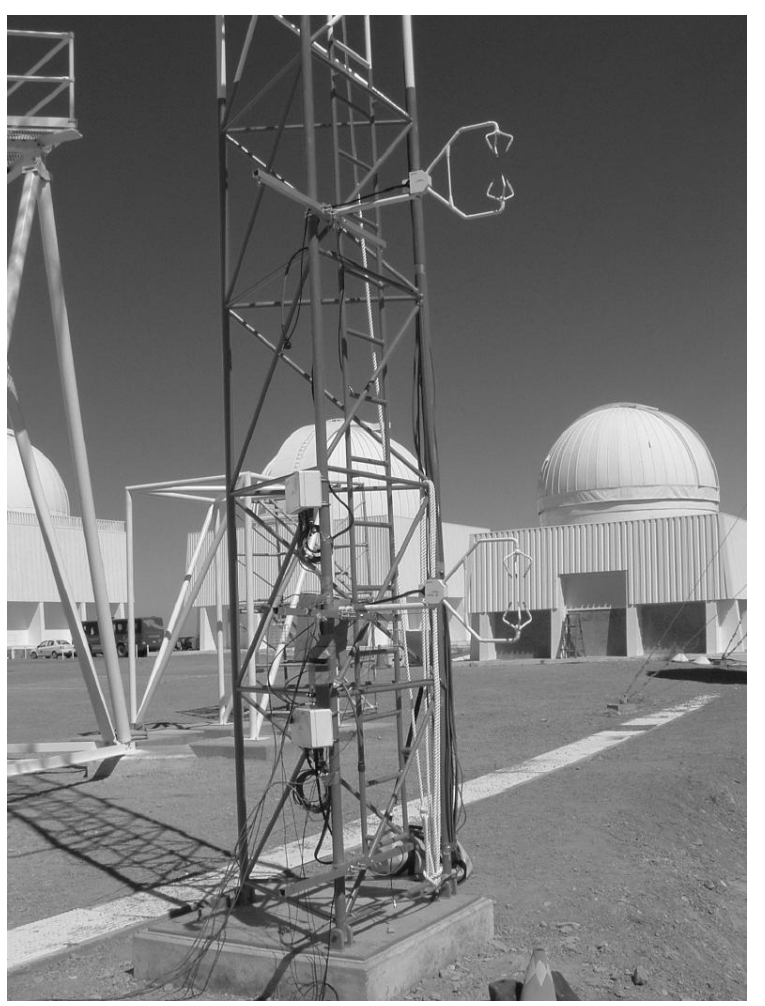

Figure 4. Probes on 30m tower during setup. Two sonic anemometers are clearly seen. Thermal probes are mounted along the horizontal arms. The sonic anemometers are normally placed at $12.4 \mathrm{~m}$ and $30 \mathrm{~m}$ altitudes.

heights (see Table 2). The instrumentation on the tower is designed to measure primarily $\mathrm{C}_{\mathrm{t}}^{2}(\mathrm{~h})$ and $\mathrm{C}_{\mathrm{n}}^{2}(\mathrm{~h})$ based on spatial and/or temporal measures of $\Delta \mathrm{T}$. We are evaluating several different types of probes in order to determine the most practical system for remote operations. The microthermal probes are a design that has been used frequently for several decades. ${ }^{1}$ The thermocouples are a new fine wire design with a fast response time ( $\sim 0.01 \mathrm{~s}$ - http://www.thermometrics.com/assets/images/thermcpl.pdf), they are wired in difference mode to a high gain amplifier. The sonic anemometers are commercial CSAT3 units that are also being used for atmospheric turbulence measurements by NCAR (http://www.atd.ucar.edu/homes/oncley/bao2004/background.pdf).

\subsection{SODARs}

SODAR devices emit pulses of high intensity sound waves and measure the returned signal due to back scattering from turbulent layers in the atmosphere. From the strength and characteristics of this returned signal the atmospheric mechanical turbulent properties and wind velocity can be found with high vertical resolution. From the mechanical turbulence it is possible to determine the optical turbulence providing a suitable temperature profile has been assumed or determined. Two SODAR (SOnic Detection And Ranging) units are being used (see Figure 5), one that covers the range from approximately $30 \mathrm{~m}$ to $800 \mathrm{~m}$ (overlapping with the MASS sampling range) and another covering $15 \mathrm{~m}$ to $200 \mathrm{~m}$ (overlapping with the $30 \mathrm{~m}$ tower).

\subsection{Portable DIMM}

A small portable DIMM system using a 12" Meade telescope and operating in the same mode as the CTIO RoboDIMM system ${ }^{5}$ is used for cross checking between instruments at different sites. The portable system can be mounted on an arm in close proximity $(\sim 1.5 \mathrm{~m})$ to our main MASS/DIMM system (see Figure 2$)$ or on a tripod for positioning close to instruments in other installations. During our comparison campaign at CTIO the portable DIMM has been mounted both next to our main system and on a special bracket inside the CTIO MASS/DIMM system dome (separation $\sim 0.9 \mathrm{~m}$ ). 


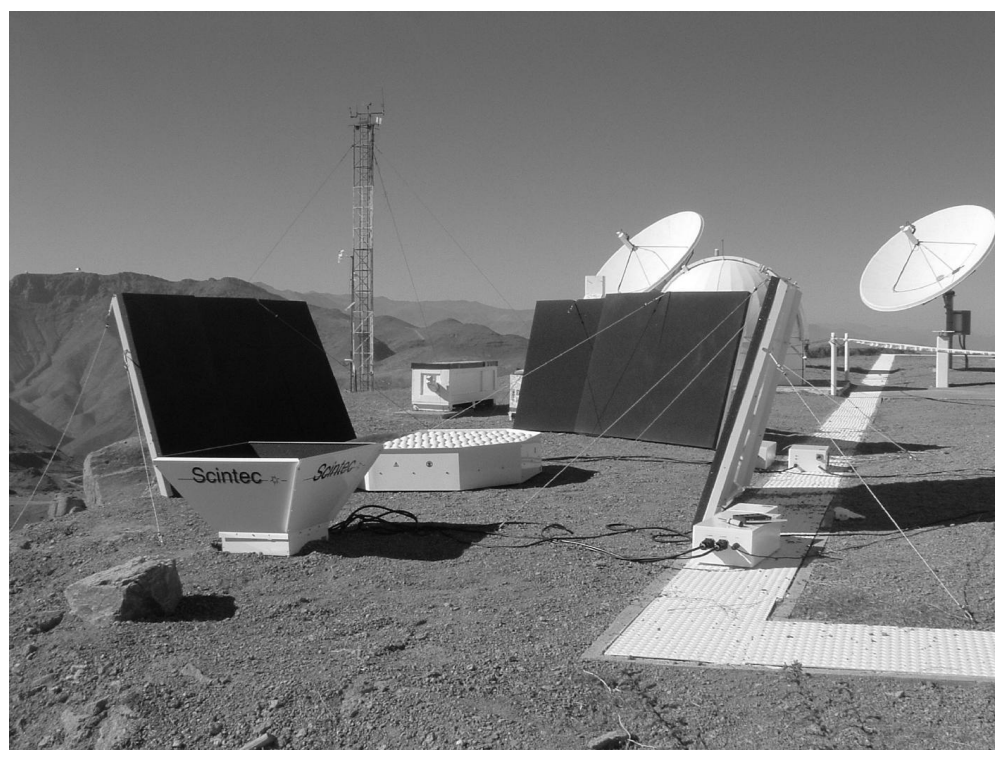

Figure 5. SODAR units during installation. Baffles are temporarily arranged to allow access. Since this photo was taken the baffles have been correctly installed (see Figure 11 to see problems of noise pollution before correct baffle installation)

\subsection{Weather station}

A small weather station is placed at each site and gathers wind speed and direction, humidity, solar irradiance, air and ground surface temperatures. The supervisor computer uses this information (along with flux information from the DIMM system) to decide whether or not to allow robotic operation.

\section{ABSOLUTE AND CROSS CALIBRATION}

\subsection{Ladder philosophy, identical equipment setup, cross checks and comparison with established methods}

We are developing a system of instrumentation that measures both the integrated effect of the whole atmosphere and the turbulence characteristics at different altitudes within the atmosphere. The different instruments have considerable overlap regions. Within these overlap regions we are striving to achieve quantitative agreement between instruments, the MASS and SODAR have an overlap region and the SODAR and tower have an overlap region. The DIMM systems measure the integrated turbulence profile, which must agree with the integral of the measured profiles.

We are aiming towards an absolute calibration of the profiling and DIMM instruments. To achieve this we are checking that the TMT DIMM and portable DIMM systems agree (the portable DIMM uses the normal accepted method for determining the seeing ${ }^{5}$ ) and that the TMT MASS agrees with the CTIO MASS/DIMM system and the CTIO portable MASS system. This process is difficult, no single instrument can be classed as a standard. Problems have been identified amongst all pieces of equipment included in the campaign and these problems are solved in an iterative manner. We are working on improving the performance and calibration of each piece of equipment until we are satisfied that it is set up properly and that the instruments' results are correct. When each piece of equipment is working correctly, the respective measurements should match those of similar instruments. Some initial results of our comparison/calibration efforts are shown below.

The portable DIMM will be used periodically for checking the calibration of remote equipment and for comparing with DIMM systems belonging to other groups. 


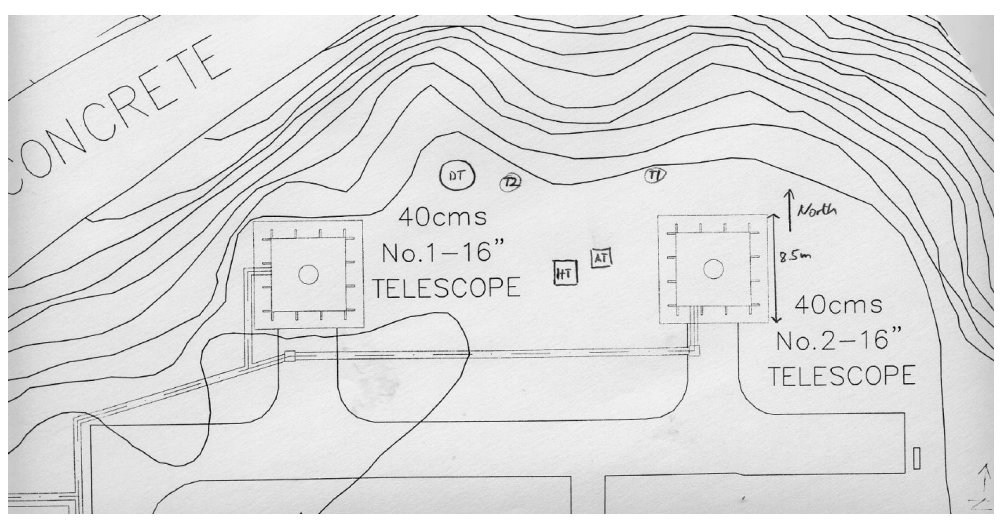

Figure 6. Layout of instruments at CTIO. Prevailing wind is from North. DT marks CTIO MASS/DIMM tower, HT and AT mark the locations of the two TMT MASS/DIMM telescope towers, T1 is the location of the 30m tower, T2 is the location of the weather station. The SODAR units are located towards the edge of the flat ground to the right of the eastern dome.

\subsection{Testing campaign at CTIO}

The aims of the CTIO campaign are to evaluate the usefulness of SODAR and a variety of tower mounted equipment for determining the structure of atmospheric turbulence profiles with regard to the requirements for TMT site selection, to investigate the absolute calibration of the different types of profiling and integrated seeing instruments, to investigate the absolute calibration between similar instruments deployed on different telescopes and to gather sufficient data to allow an evaluation of computational fluid dynamics models of the atmospheric turbulence.

The locations of the equipment at CTIO are shown in Figure 6. The CTIO campaign has included amongst other things;

- Setting up the TMT MASS/DIMM system on two 6.5m telescope towers (equivalent to height of the CTIO MASS/DIMM system) at distance of approximately $10 \mathrm{~m}$ and $15 \mathrm{~m}$ from the CTIO MASS/DIMM tower).

- Installation of a large and a small SODAR unit at a distance of about $20 \mathrm{~m}$ from the telescope towers.

- Erection of a 30m tower about 8m away from the telescope towers and 15m away from the SODARS.

- Mounting of the portable DIMM alongside the TMT MASS/DIMM unit and then alongside the CTIO MASS/DIMM unit.

- The running of a portable MASS unit in the western most dome in Figure 6.

- Gathering of microthermal and SODAR measurements simultaneous with upwind balloon launches.

At the time of writing the campaign at CTIO is underway and incomplete. The comparison between the MASS and DIMM units has produced very convincing results. Work on the $30 \mathrm{~m}$ tower and SODAR comparisons is still underway, quantitive agreements are expected.

\subsubsection{Results from the CTIO comparison and development campaign}

There is agreement between TMT \& portable DIMM systems when mounted next to each other and the CTIO and portable DIMM systems when next to each other, see Figure 7. Differences between the CTIO and TMT DIMMs are variable but are generally small $(0.0 "-0.2 ")$, the TMT and CTIO systems are physically separated by about $10 \mathrm{~m}-15 \mathrm{~m}$ and differences in local turbulence are probably able to explain the discrepancies in seeing values. This is being investigated by studying the correlations between wind speed \& direction and discrepancy.

The TMT and CTIO MASS systems give the same measurements to within the level of measurement uncertainty, see Figures 3 and 8. The CTIO portable MASS was also used for several nights and again gave extremely consistent results with the other two MASS systems. 

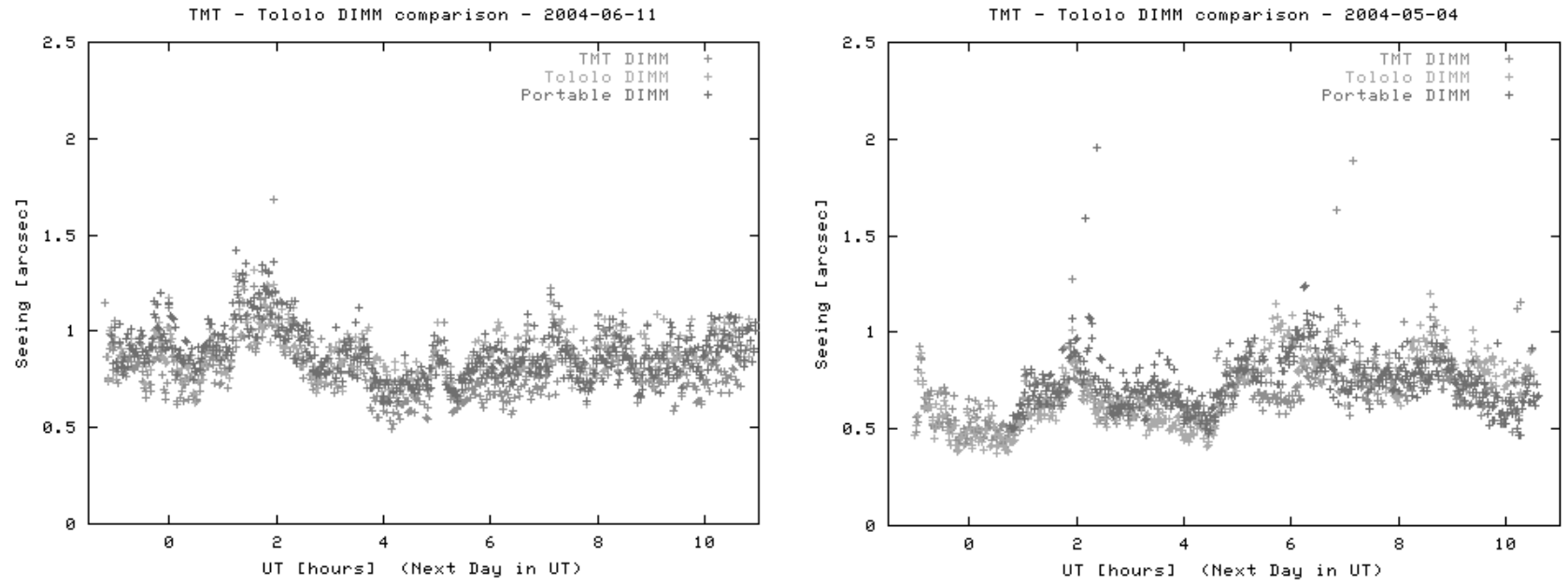

Figure 7. Comparison of DIMM instruments. Left: With portable DIMM (black points) next to CTIO MASS/DIMM (light gray). Note excellent agreement between CTIO and portable systems with occasional discrepancies against the TMT DIMM (dark gray). Right: Now portable DIMM is next to TMT MASS/DIMM, discrepancies sometimes occur between CTIO and TMT+portable systems. These results indicate the significance of local effects.

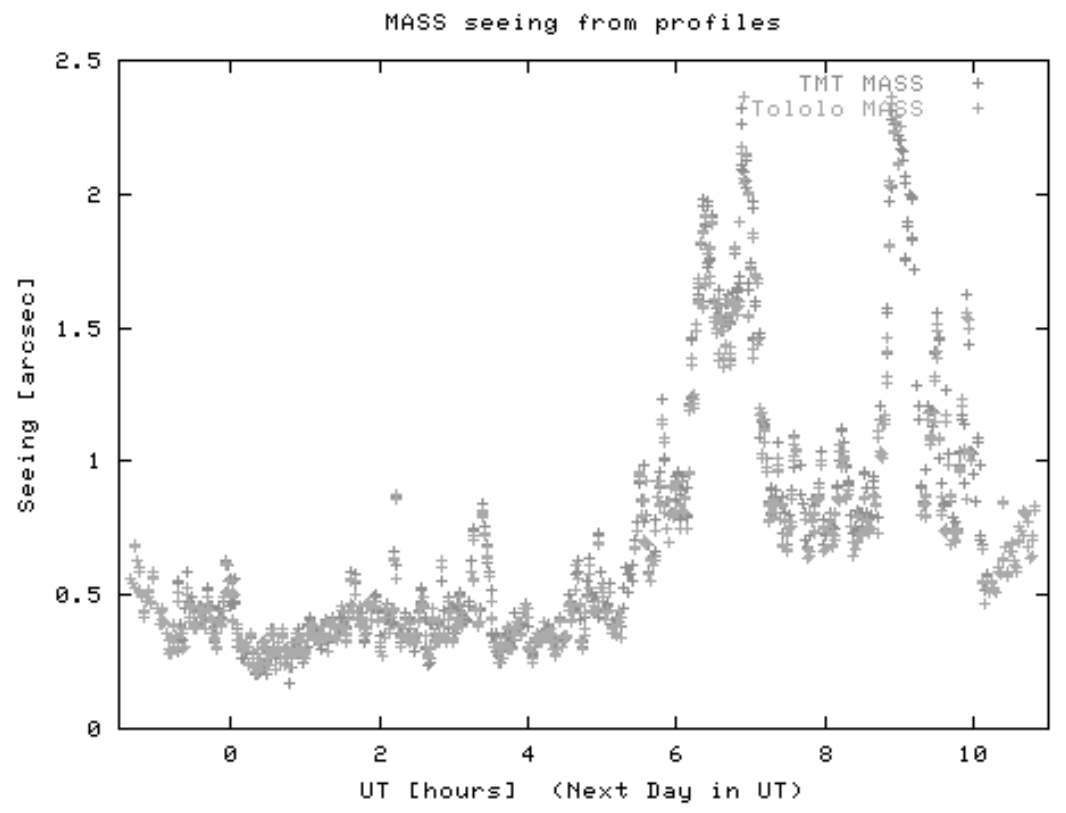

Figure 8. Comparison of the seeing predicted for the $\mathrm{C}_{\mathrm{n}}^{2}$ profiles from the CTIO (gray points) \& TMT (black points) MASS systems. Note the excellent agreement, the black points are covered by the grey points 


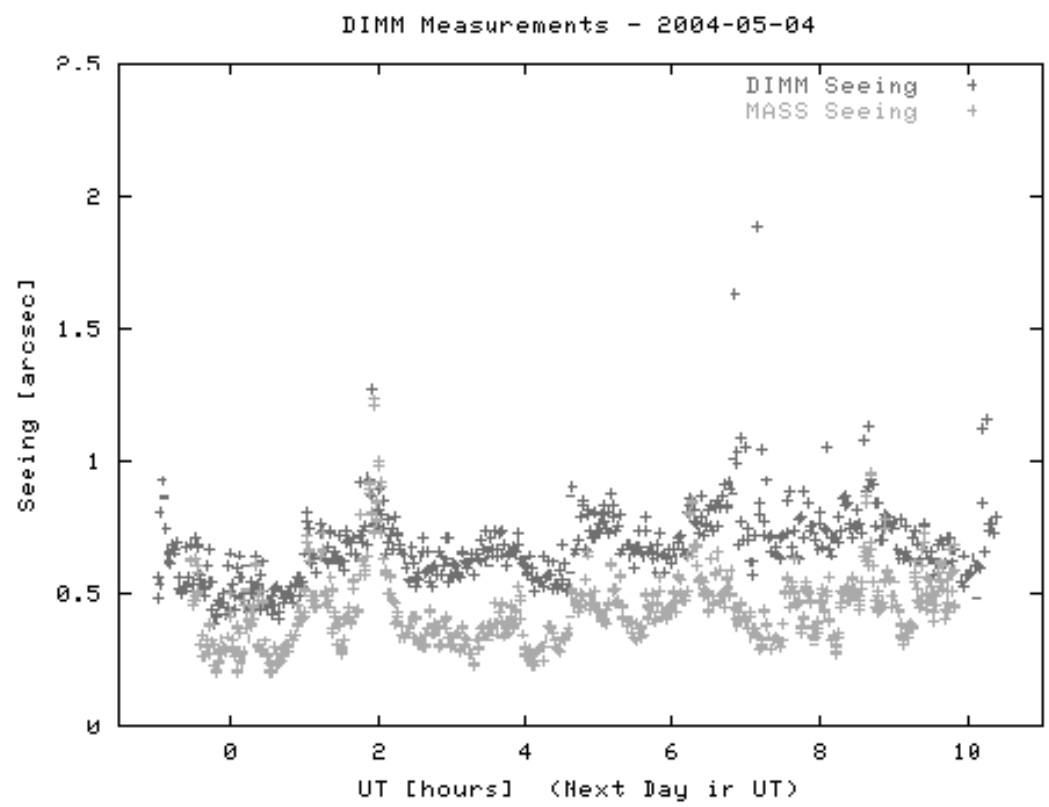

Figure 9. DIMM seeing \& calculated MASS seeing, the difference between the two values indicates the component of the seeing due to the lower few hundred metres of the atmosphere
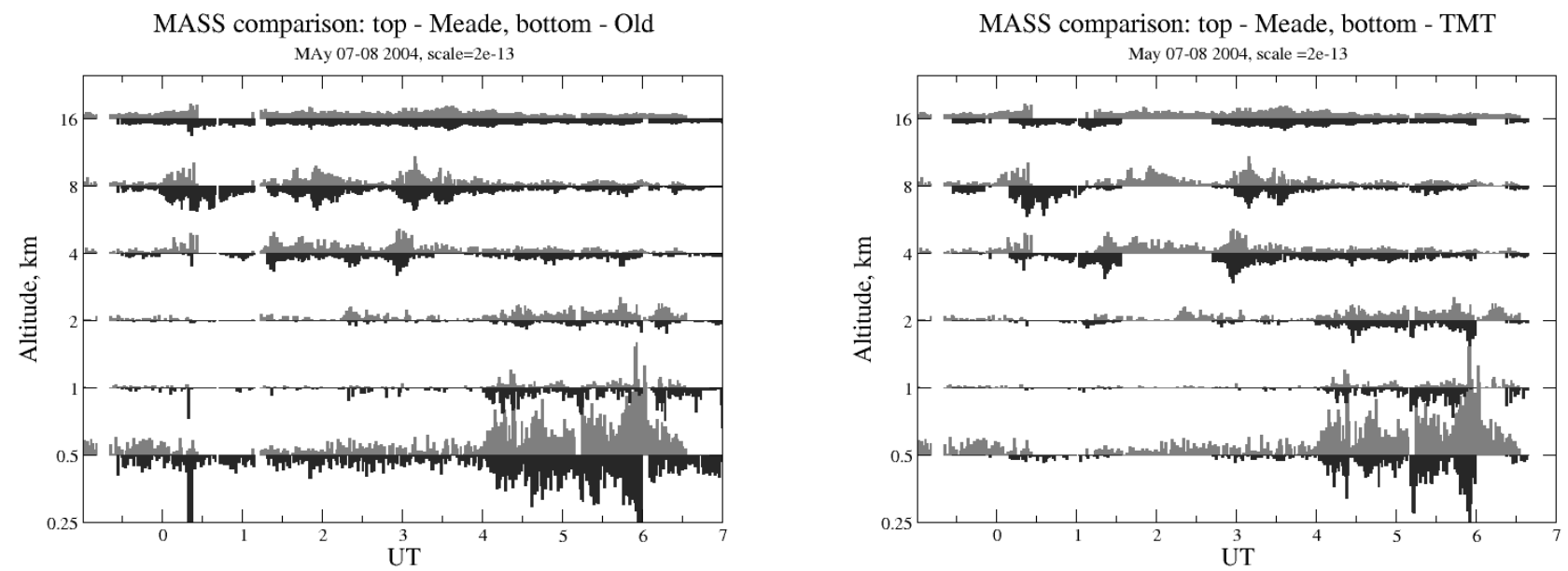

Figure 10. Left: $\mathrm{C}_{\mathrm{n}}^{2}$ profiles from the CTIO and portable MASS units. Right: $\mathrm{Cn}^{2}$ profiles from TMT and CTIO MASS instruments 


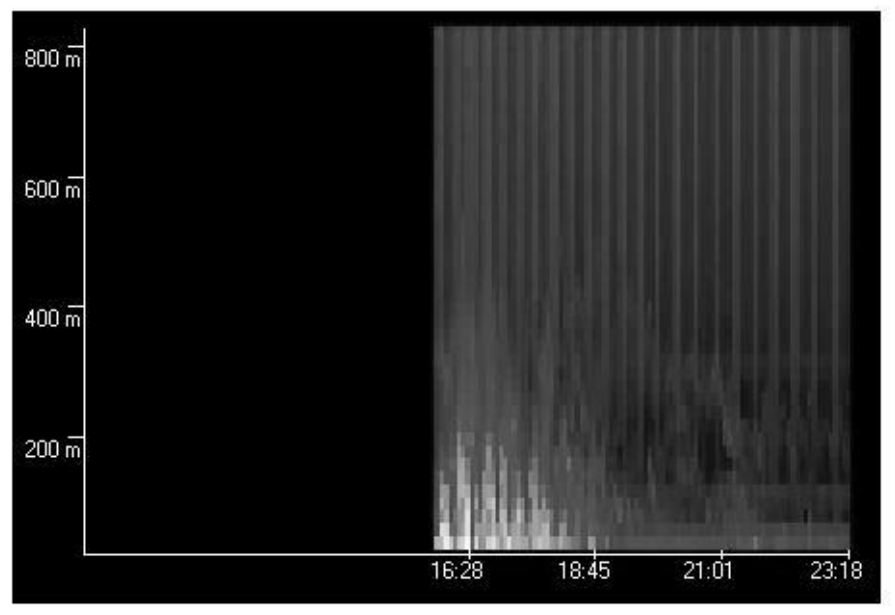

Figure 11. SODAR profiles showing reducing turbulence around sunset. The regular vertical stripe pattern is due to sound contamination by a nearby air conditioning unit, this problem has now been cured.
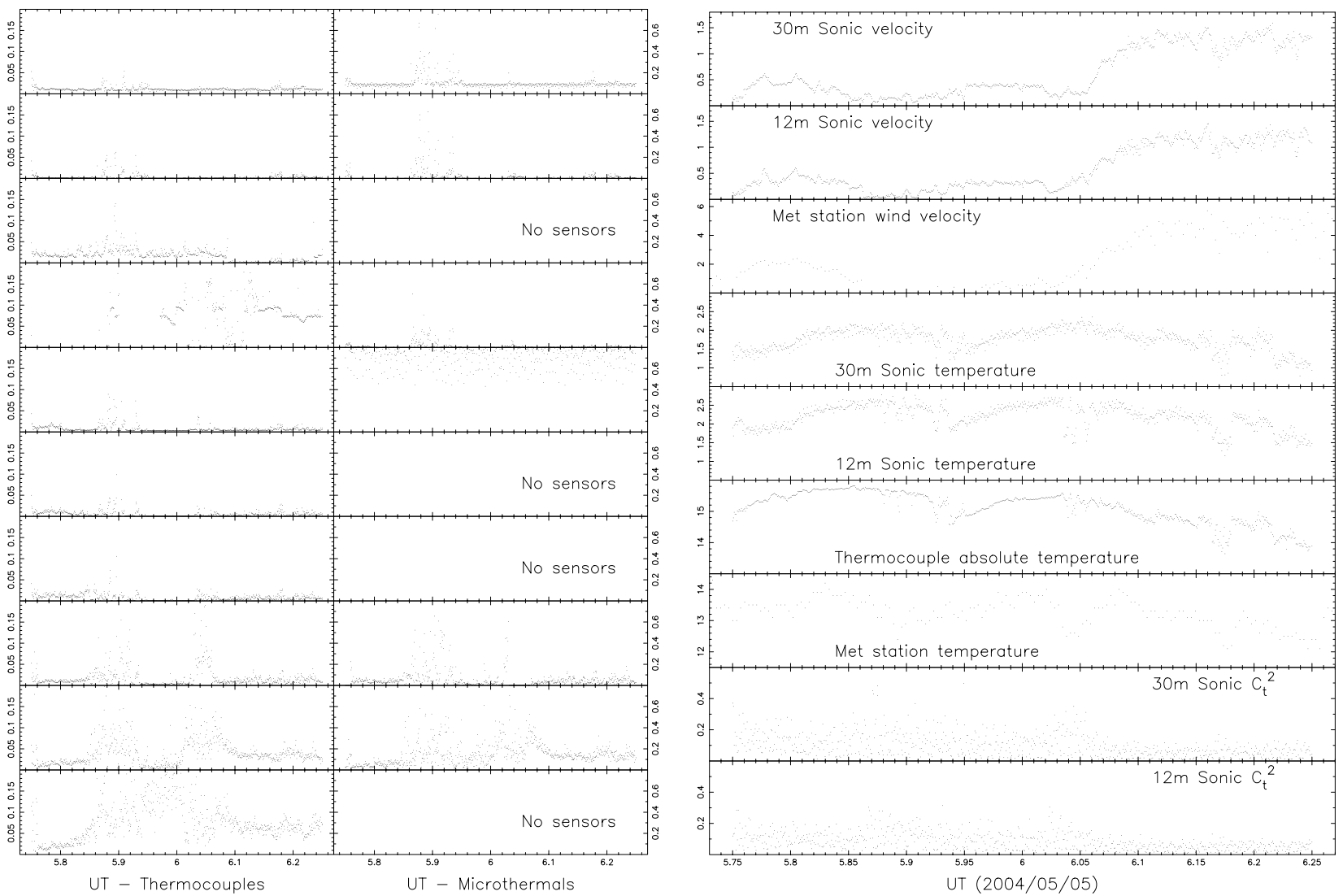

Figure 12. Left: Thermocouple \& microthermal probe $C_{t}^{2}$ measurements show qualitative agreement at some altitudes, levels 4 and 5 have high external noise problems, level 1 (top, 30m) has some low level problems with noise. Right: Comparison between the Sonic anemometer, ground weather station and absolute temperature thermocouple at level 5 . The bottom two panels are the levels 1 and $5 \mathrm{C}_{\mathrm{t}}^{2}$ values from the sonic anemometers, the large noise problems are evident. 
Many factors causing discrepancies between the TMT and CTIO MASS/DIMM systems have been discovered and solved. For example, the airflow through and around the CTIO telescope has been improved by the addition of vents in the tower and fully opening the dome when the wind speed is low enough for the Meade telescope to operate, the plate scale of the DIMM systems have been properly calibrated and problems due to the inability of the Meade telescopes to maintain accurate focus have been solved by implementing strict operating procedures.

The large SODAR shows a rough qualitative agreement with the MASS \& 30m tower in as much that when the difference between the MASS and DIMM measurements are large (indicating large low altitude turbulence) then the $\mathrm{C}_{\mathrm{t}}^{2}$ profile from the SODAR shows high turbulence levels. At this time there has been no in-depth investigation of the SODAR measurements. An example of measurements from the large SODAR are shown in Figure 11. Problems due to noise pollution and echoes have been solved by proper baffling arrangements.

The thermal probes on the $30 \mathrm{~m}$ tower show almost proper quantitative agreement in the case of $\mathrm{C}_{\mathrm{t}}^{2}$ for the differenced thermocouples and microthermal probes and absolute temperature from the sonic anemometers and absolute temperature thermocouples, see Figure 12. Further work is needed to extract proper $\mathrm{C}_{\mathrm{t}}^{2}$ values from the sonic anemometer measurements, to reduce the calibration differences between the different $\mathrm{C}_{\mathrm{t}}^{2}$ instruments and to get proper values of the absolute temperature.

\section{FUTURE WORK}

The development \& characterisation campaign will be completed by the end of August 2004. The SODAR and $30 \mathrm{~m}$ tower calibration is as yet unfinished. We will also deploy a second TMT MASS/DIMM system along side the unit presently installed at CTIO to prove that identical physical systems are strictly comparable.

The final operational configuration of the $30 \mathrm{~m}$ tower will be determined by the conclusion of the above campaign.

We need to deploy full site testing systems at the remaining candidate sites and upgrade our first remote system.

Analysis and interpretation of the incoming data is a high priority and new personnel will spear head this effort as the data flow will increase several times above its present rate.

\section{ACKNOWLEDGMENTS}

The Thirty Meter Telescope (TMT) Project is a partnership of the Association of Universities for Research in Astronomy (AURA), the Association of Canadian Universities for Research in Astronomy (ACURA), the California Institute of Technology and the University of California. The partners gratefully acknowledge the support of the Gordon and Betty Moore Foundation, the US National Science Foundation, the National Research Council of Canada, the Natural Sciences and Engineering Research Council of Canada, and the Gemini Partnership.

We thank the many personnel, particularly the CTIO staff, that have assisted with the development and deployment of the TMT site testing equipment and whose help has been and will continue to be vital for this project.

\section{REFERENCES}

1. F. Forbes, D. Morse \& G. Poczulp, "Site survey instrumentation," 1986, SPIE 628, p. 118

2. M. Sarazin \& A. Tokovinin, "The Statistics of Isoplanatic Angle and Adaptive Optics Time Constant derived from DIMM Data," in Beyond Conventional Adaptive Optics, ESO conf. proc. Vol. 58, ed. E. Vernet et al., pp. $321,2002$.

3. A. Tokovinin, "From Differential Image Motion to Seeing," PASP, vol. 114, pp. 1156, 2002.

4. A. Tokovinin \& V. Kornilov, "Measuring turbulence profile from scintillations of single stars," in Astronomical Site Evaluation in the visible and Radio Range, ASP Conf. Ser., eds. Z. Benkhaldoun, C. Muñoz-Tuñon, J. Vernin, 2001.

5. J. Vernin \& C. Munoz-Tunon, 1995, PASP, vol. 107, p. 265

6. K. Vogiatzis \& D. De Young, SPIE Proc. Backaskog Castle, Sweden, 2003 